\title{
Gene Expression Identifies Distinct Ascending Glutamatergic Pathways to Frequency-Organized Auditory Cortex in the Rat Brain
}

\author{
Douglas A. Storace, ${ }^{1,2}$ Nathan C. Higgins, ${ }^{1}$ Jennifer A. Chikar, ${ }^{2}$ Douglas L. Oliver, ${ }^{2}$ and Heather L. Read ${ }^{1,3}$ \\ ${ }^{1}$ Department of Psychology, Behavioral Neuroscience Division, University of Connecticut, Storrs, Connecticut 06269, ${ }^{2}$ Department of Neuroscience, \\ University of Connecticut Health Center, Farmington, Connecticut 06030, and ${ }^{3}$ Biomedical Engineering, University of Connecticut, Storrs, Connecticut \\ 06269
}

A conserved feature of sound processing across species is the presence of multiple auditory cortical fields with topographically organized responses to sound frequency. Current organizational schemes propose that the ventral division of the medial geniculate body (MGBv) is a single functionally homogenous structure that provides the primary source of input to all neighboring frequency-organized cortical fields. These schemes fail to account for the contribution of $\mathrm{MGBv}$ to functional diversity between frequency-organized cortical fields. Here, we report response property differences for two auditory fields in the rat, and find they have nonoverlapping sources of thalamic input from the MGBv that are distinguished by the gene expression for type 1 vesicular glutamate transporter. These data challenge widely accepted organizational schemes and demonstrate a genetic plurality in the ascending glutamatergic pathways to frequency-organized auditory cortex.

\section{Introduction}

The ability to discriminate sound sources in natural scenes relies on distinct sound processing properties of different frequencyorganized auditory cortices in mammals (Polley et al., 2006; Lomber and Malhotra, 2008; Nodal et al., 2010), but the mechanisms underlying these functional differences remain a mystery. By definition, "core" auditory cortices have a fine-grain topographic organization of responses to sound frequency and receive their primary thalamic input from the ventral division of the medial geniculate body (MGBv) of the thalamus (Kaas and Hackett, 2000). Several mammals including humans have multiple "core" fields outside the primary (A1) auditory cortical field (Formisano et al., 2003; Schreiner and Winer, 2007; Hackett, 2011). Gerbils, guinea pigs, and rats have overlapping hearing ranges and similar frequency organization of A1 relative to the anterior auditory field (AAF) and a third ventral field with distinct frequency organization (Wallace et al., 2000; Kalatsky et al., 2005; Donishi et al., 2006; Budinger and Scheich, 2009). In the

\footnotetext{
Received March 8, 2012; revised Aug. 7, 2012; accepted Sept. 6, 2012.

Author contributions: D.A.S., D.L.O., and H.L.R. designed research; D.A.S., N.C.H., J.A.C., and H.L.R. performed research;D.A.S., N.C.H., D.L.O., and H.L.R. contributed unpublished reagents/analytic tools; D.A.S., N.C.H., and H.L.R. analyzed data; D.A.S., J.A.C., D.L.O., and H.L.R. wrote the paper.

This work was supported by National Institute of Child and Health Development Grant HD2080 (H.L.R.), National Institutes of Health Grant DCO00189 (D.L.O.), and an intramural University of Connecticut Grant (H.L.R.). We thankA. Burkhalter and A. Reyes for comments on an early draft of this manuscript.

Correspondence should be addressed to Heather L. Read, Department of Psychology, Behavioral Neuroscience Division, University of Connecticut, 406 Babbidge Road, Unit 1020, Storrs, CT 06269. E-mail: heather.read@uconn.edu.

D. A. Storace's present address: Department of Cellular and Molecular Physiology, Yale University, New Haven, CT 06520.

DOI:10.1523/JNEUROSCI.1310-12.2012

Copyright $\odot 2012$ the authors $\quad 0270-6474 / 12 / 3215759-10 \$ 15.00 / 0$
}

rat, this ventral field is called the suprarhinal auditory field (SRAF) (Polley et al., 2007). Neurons in caudal SRAF (cSRAF) respond more selectively to sound frequency and position cues than their frequency-matched counterparts in A1 (Polley et al., 2007; Higgins et al., 2010). These sound-encoding differences could stem from physiologic and anatomic differences evident along the caudal-rostral axis of MGBv (Redies et al., 1989; Rodrigues-Dagaeff et al., 1989; Cant and Benson, 2007; Storace et al., 2011). However, at present there are no known anatomic or molecular indices that subdivide $\mathrm{MGBv}$ along its caudal-torostral axis (Morest, 1964; Clerici and Coleman, 1990; Winer et al., 1999a, 2005; Storace et al., 2010).

The majority of the principal neurons in MGBv likely release glutamate neurotransmitter as they express the gene and corresponding mRNA encoding type 2 vesicular glutamate transporter (VGLUT2) which in turn is critical for loading glutamate into synaptic vesicles (Fremeau et al., 2004a; Barroso-Chinea et al., 2007; Hackett et al., 2011; Ito et al., 2011). This expression pattern is consistent with a VGLUT2 protein-dense terminal field found within thalamic recipient layers of sensory cortex (Kaneko et al., 2002; Nahmani and Erisir, 2005; Graziano et al., 2008; Hackett and de la Mothe, 2009). However, recent studies find that type 1 glutamate transporter (VGLUT1) mRNA is coexpressed with VGLUT2 mRNA in a subset of MGBv neurons in rodents (Barroso-Chinea et al., 2007; Ito et al., 2011). This raises the question of whether MGBv pathways to different frequencyorganized cortical fields have different gene expression patterns for glutamate transporter type.

Here, we identify sound response differences between adjacent frequency-organized cortices A1 and cSRAF in the rat. We confirm that the MGBv provides the majority of inputs to a tar- 
geted injection layer in both fields. Furthermore, we find that $\mathrm{MGBv}$ neurons that project to A1 express VGLUT1 mRNA, whereas those that project to CSRAF do not. These findings offer a novel explanation and molecular marker for functional diversity of ascending sensory pathways to frequency-organized auditory cortices.

\section{Materials and Methods}

Surgery and imaging in adult rats. Animals were housed and handled according to a protocol approved by the Institutional Animal Care and Use Committee (University of Connecticut, Storrs). Intrinsic imaging was performed on 28 male rats with a mean age and weight of $71 \pm 4 \mathrm{~d}$ and $214 \pm 14 \mathrm{~g}$, respectively. Each of these rats was used in one or more additional experiments that include retrograde tracer injections, in situ hybridization, unit recordings, and immunohistochemical analysis. Anesthesia was induced with a mixture of ketamine $(50 \mathrm{mg} / \mathrm{kg}$ ), xylazine ( 5 $\mathrm{mg} / \mathrm{kg})$, and acepromazine $(2.5 \mathrm{mg} / \mathrm{kg})$, and was maintained in a stage III, plane II of general anesthesia with sodium pentobarbital $(25 \mathrm{mg} / \mathrm{kg}$, i.p.) for surgical and experimental procedures. Anesthesia was supplemented as needed to maintain areflexia, and anesthetic depth was monitored periodically via pedal reflex and heart rate (electrocardiogram, GE Healthcare Life Sciences). Tracheotomy and cisterna magnum puncture were performed and atropine sulfate $(0.1 \mathrm{mg} / \mathrm{kg})$ and dexamethasone $(0.25 \mathrm{mg} / \mathrm{kg})$ were administered to minimize respiratory noise and cerebral edema. The skull and dura were removed to expose the temporal cortex. The exposure was covered with agar and sealed with a glass coverslip for optical imaging of intrinsic metabolic responses.

Sound stimuli, delivery, and design. All sounds were presented to both ears through hollow ear-bars via custom modified (Beyer DT 770) speakers calibrated for frequencies in the range of $1-45 \mathrm{kHz}( \pm 3 \mathrm{~dB})$ and linearity (input/output coherence $>0.95$ ) in a closed system with a 400 tap FIR inverse filter implemented on a Tucker Davis Technologies (TDT) RX6 multifunction processor. Auditory stimuli used for multiunit recordings were flat spectrum noise tokens $(<5 \mathrm{~ms}$ rise time, $50 \mathrm{~ms}$ duration) delivered via a TDT RX6 multifunction processor at $96 \mathrm{kHz}$ (Higgins et al., 2010). Auditory stimuli used for Fourier intrinsic imaging were a continuous sequence of 16 pure tones ( $50 \mathrm{~ms}$ duration, $5 \mathrm{~ms}$ rise and decay time, $250 \mathrm{~ms}$ interstimulus interval) from 2 to $32 \mathrm{kHz}(0.25$ octave steps) with a $4 \mathrm{~s}$ period in ascending or descending order (Kalatsky et al., 2005; Polley et al., 2007; Higgins et al., 2010; Storace et al., 2010, 2011) delivered via a Lynx Studio Technology professional audio card at $98 \mathrm{kHz}$.

Fourier optical imaging. Fourier optical imaging was used to map tone frequency response organization in auditory cortex. Images were acquired with a Dalsa $1 \mathrm{M} 30 \mathrm{CCD}$ camera with a $512 \times 512$ pixel array covering a $4.6 \times 4.6 \mathrm{~mm}^{2}$ area (Kalatsky et al., 2005; Polley et al., 2007; Higgins et al., 2010; Storace et al., 2010, 2011). Surface vascular patterns were imaged with a green $(546 \mathrm{~nm})$ interference filter at a $0 \mu \mathrm{m}$ plane of focus. Intrinsic activity was imaged at a plane of focus of $600 \mu \mathrm{m}$ below the surface blood vessels using a red $(610 \mathrm{~nm})$ interference filter. Ascending and descending tone sequences yielded similar frequency topographies in all regions; thus, hemodynamic delay was corrected by subtracting the ascending and descending frequency phase maps to generate a difference phase map (Kalatsky et al., 2005). The optical best frequency $(\mathrm{BF})$ was the value obtained from single pixels at designated positions in the optical difference phase map. The locations of A1, cSRAF, rostral SRAF (rSRAF), and ventral (VAF), posterior (PAF), and anterior auditory field (AAF), were determined physiologically by a change in the direction of the frequency gradient observed in the optical frequency map as described in detail previously (Higgins et al., 2010). Post hoc histochemistry confirmed that the dorsal border of Al and the ventral border of cSRAF fall within the cytoarchitectonically defined temporal area 1, Te1 (LeDoux et al., 1985; Paxinos and Watson, 1997).

Temporal multiunit response analysis. Multiunit responses were recorded in A1 and $\operatorname{cSRAF}\left(N_{\mathrm{A} 1}=159 ; N_{\mathrm{cSRAF}}=91\right)$ in response to white noise bursts presented at varying interaural level differences (ILDs) and average binaural levels (ABLs). The dataset range was limited in the ABL dimension from threshold to $40 \mathrm{~dB}$ above threshold, and best ILD \pm 5
$\mathrm{dB}$. Analyses for the calculation of best ILD and ABL thresholds have been previously detailed (Higgins et al., 2010). The poststimulus time histogram (PSTH) was generated from the limited response range and used to find the maximum amplitude, time to onset and time to peak. Rising and falling slopes were identified by fitting the PSTH with an $\alpha$ function of the form:

$$
A *\left(1-e^{-\left(t-t_{0}\right) / T_{\text {Rise }}}\right) * e^{-\left(t-t_{0}\right) / T_{\text {Decay }}}
$$

where $A$ is the magnitude, $t$ is time, $t_{0}$ is the time of significant onset, $T_{\text {rise }}$ is the time from onset to peak, and $T_{\text {decay }}$ is the time from peak to offset. Times to onset and offset were determined as the first and second crossings of a set significance threshold $(\alpha=0.05)$ (Escabí et al., 2007), and were used to determine the significant portion of the PSTH to model. The best fit function for each unit was calculated using the lsqcurvefit MATLAB function (MathWorks), and cross validation was used to assess quality of fit. Each stimulus was presented 16 times, therefore the model was based on eight trials, then validated by comparison to the other eight trials. This procedure was repeated 500 times for each unit with a random selection of trials designated in each iteration. A subset of the data was analyzed over 1000 iterations of the cross validation procedure and no significant differences were observed.

Retrograde tracer and VGLUT2 histology. Following optical imaging, the retrograde tracers Cholera toxin B (CTB, $10 \mu \mathrm{g} / \mu \mathrm{l}$ List Biological Laboratories) and CTB conjugated with gold (CTBG, $10 \mu \mathrm{g} / \mu \mathrm{l}$ List Biological Laboratories) were injected (Nanoliter 2000, World Precision Instruments) using a glass capillary (tip diameter $10-12 \mu \mathrm{m})$ into frequency-matched $\left(N_{\mathrm{A} 1}=8\right.$, $\mathrm{BF}_{\mathrm{A} 1}=8.4 \pm 0.6 \mathrm{kHz}, N_{\mathrm{cSRAF}}=8, \mathrm{BF}_{\mathrm{SRAF}}=7.3 \pm 0.4 \mathrm{kHz}, t_{(16)}=1.56, p=$ $0.14)$ contours in Al and cSRAF. Injections targeted layer IV in both areas and had a cortical surface separation of $1.8 \pm 0.05 \mathrm{~mm}$. Tracer transport occurred during an $\sim 24 \pm 0.5 \mathrm{~h}$ survival time under anesthesia. For experiments in which VGLUT2 protein expression was analyzed, a similar procedure was used except that green and red fluorescent beads (undiluted, Lumafluor) were injected into A1 and $\operatorname{cSRAF}(N=4)$ for identification purposes. Rats were given a lethal dose of pentobarbital and perfused transcardially with $4 \%$ paraformaldehyde. Brains were removed, blocked (Model PA 001 Rat Brain Blocker, Kopf Instruments), and postfixed in 4\% paraformaldehyde. Following postfixation, the brains were cut at $50 \mu \mathrm{m}$ on a vibratome $(N=2$; retrograde tracer experiments) (VT1000, Leica Microsystems), or cryoprotected in diethylpyrocarbonate-treated $30 \%$ sucrose in phosphate buffer for at least $3 \mathrm{~d}$, and cut at $40 \mu \mathrm{m}(N=7$; retrograde tracer and in situ experiments) or $20 \mu \mathrm{m}(N=4$; VGLUT2 immunohistochemistry experiments) on a freezing microtome (HM440E, Microm International). CTB label (CTB-IGG, List Biological Laboratories) and VGLUT2 protein (Synaptic Systems, catalog \#135 402) were detected using immunohistochemistry, and CTBG was detected via silver enhancement (KPL Labs). All rats that received dual CTB and CTBG tracer injections had at least one series of tissue double-reacted for both tracers. In cortex, locations of Tel and temporal areas 2 and 3 (Te2 and Te3, respectively) were determined from cytoarchitectonics of Nissl-reacted and immunoreacted sections (LeDoux et al., 1985; Paxinos and Watson, 1997). Photomicrographs and drawings of histological sections were prepared using Adobe Photoshop and Illustrator (Adobe Systems) and were contrast enhanced, brightened, and color balanced.

Retrograde tracer analysis. Retrograde tracer injection deposit volumes and locations did not overlap and were measured in Stereo Investigator (MBF Bioscience) using the Cavalieri estimator. Injection deposits had an average caudal-rostral spread of $600 \pm 52 \mu \mathrm{m}(N=14)$. Injection deposit volumes for A1 and cSRAF were $0.13 \pm 0.03(N=7) \mathrm{mm}^{3}$ and $0.2 \pm 0.02$ $(N=7) \mathrm{mm}^{3}$, respectively, and were not significantly different $\left(t_{(12)}=0.27\right.$, $p=0.79)$. The distance from the pia to the center of each injection site was $512 \pm 27(N=13) \mu \mathrm{m}$. The injection deposits fell within the boundaries of $\mathrm{A} 1$ and CSRAF, and were located $3.4 \pm 0.09(N=6)$ and $1.7 \pm 0.06(N=7)$ $\mathrm{mm}$ dorsal to the nadir of the rhinal fissure, respectively. Two experiments were excluded from injection volume and distance analysis as their cortex was removed and cut differently for a separate experiment. All histological distance and area measurements were taken directly from the sections.

Bright-field in situ hybridization histochemistry. Digoxigenin (DIG)labeled sense and antisense riboprobes were made from the cDNAs of 
A
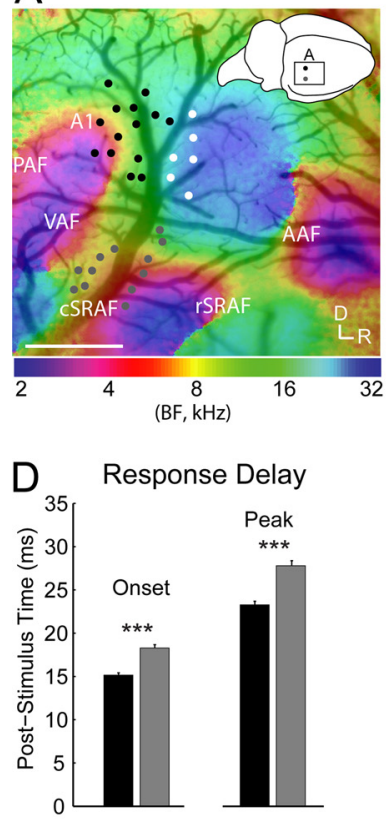

B

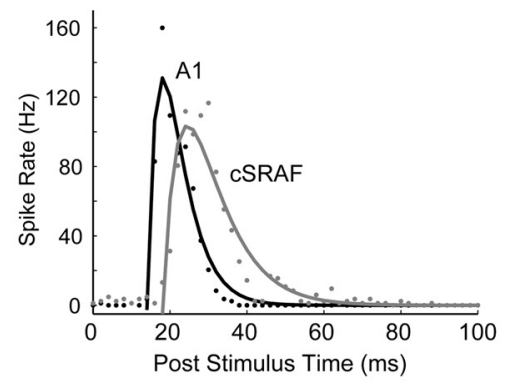

C

Response Magnitude

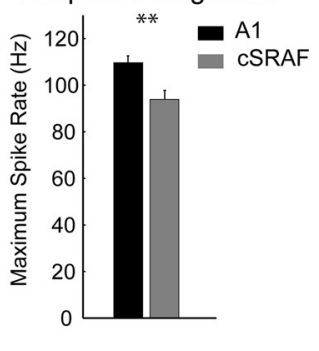

$\mathrm{E}$

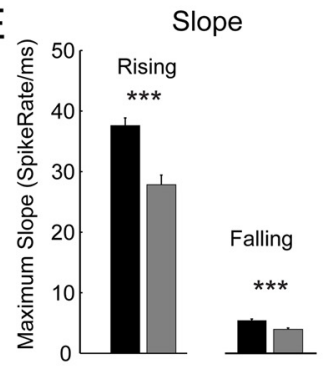

$\mathrm{F}$

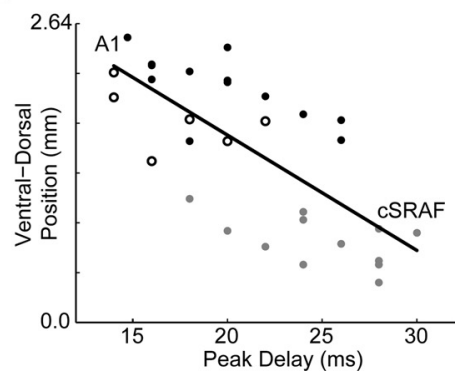

Figure 1. A1 and CSRAF have spatially nonoverlapping sound frequency response organization and distinct time course and magnitude responses to noise stimuli. $A, 0 p t i c a l$ imaging reveals intrinsic metabolic responses to tone sequences. Color indicates BF at that pixel. The BF gradient directions were used to locate A1, VAF, CSRAF, rSRAF, PAF, and AAF (Higgins et al., 2010). Positions for recording the spike rate responses to noise indicated with black (A1), gray (CSRAF), and white (A1, higher BF) circles. Inset indicates the approximate area of the recording window with the centers of $A 1$ and CSRAF marked with circles. $B$, Exemplar regional poststimulus time spike rate responses to noise; fits to the corresponding data. $C$, Mean response magnitudes were larger in $A 1$ ( $A 1: 110 \pm$ 37 spikes/s, CSRAF: $94 \pm 37$ spikes $/ s ; p<0.01)$. D, Mean response delays were shorter in A1 $\left(t_{1}: A 1=15 \pm 3 \mathrm{~ms}\right.$, CSRAF $=18 \pm 4 \mathrm{~ms}, p<0.001, t_{\mathrm{p}}: A 1=23 \pm 5 \mathrm{~ms}$, cSRAF $=28 \pm 6 \mathrm{~ms}, p<$ 0.001). $\boldsymbol{E}$, Mean slopes of the response were larger in A1 (Rising: $\mathrm{A} 1=36 \pm 16$ spike rate/ms, CSRAF $=26 \pm 15$ spike rate $/ \mathrm{ms}, p<0.001$, Falling: $\mathrm{A} 1=5 \pm 3$ spike rate $/ \mathrm{ms}, \mathrm{CSRAF}=4 \pm 2$ spike rate/ms, $p<0.001)$. $\boldsymbol{F}$, Response delays increased with ventral position shifts from A1 to CSRAF $(r=0.7, p<0.001)$. Data in $C-E$ are means from 250 recording sites in 15 rats; error bars indicate SEM. All $p$-values are the result of two-tailed $t$ tests. The positions of auditory cortical fields are labeled as defined in this and subsequent figures. $S$ cale bar, $1 \mathrm{~mm}$.
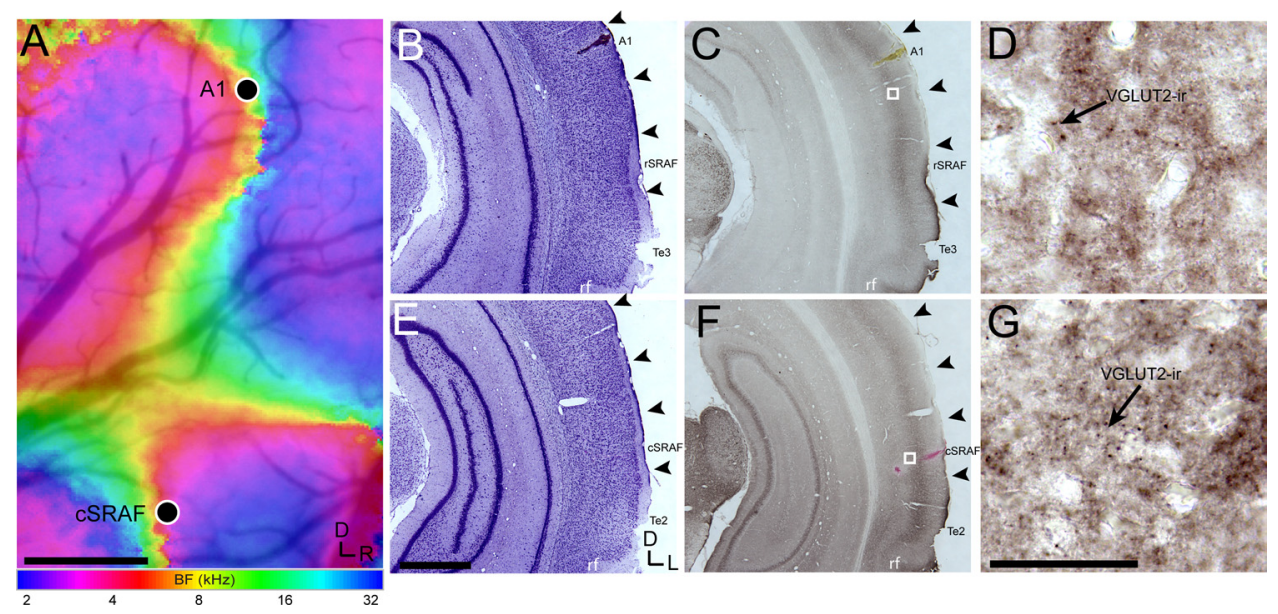

Figure 2. A1 and CSRAF both contain high concentrations of VGLUT2 protein in the middle cell layers III and IV, whereas Te3 does not. $A$, Optical intrinsic imaging was used to map the frequency response organization of the auditory cortex to guide fluorescent bead injections (circles) into A1 and CSRAF. B, E, Nissl-reacted sections demonstrate the location and cytoarchitectonics of both regions with arrows indicating the estimated boundaries between cortical fields. $C, F$, Sections immunoreacted for VGLUT2 demonstrate dense VGLUT2 protein expression in the middle cortical layers. $\boldsymbol{D}, \mathbf{G}$, High-magnification photomicrographs from $\boldsymbol{C}$ and $\boldsymbol{F}$ (white boxes) demonstrate VGLUT2 terminals (VGLUT2-ir). Scale bars: $\boldsymbol{A}, 0.5 \mathrm{~mm} ; \boldsymbol{B}, \boldsymbol{C}, \boldsymbol{E}, \boldsymbol{F}, 1 \mathrm{~mm} ; \boldsymbol{D}, \boldsymbol{G}, 0.05 \mathrm{~mm}$. The region labeled ISRAF in $B$ and $C$ is near the CSRAF and rSRAF border. Position of the rhinal fissure is labeled " $r f$ " in this and subsequent figures. VGLUT2-ir, VGLUT2 immunoreactive.

mouse VGLUT1 (nucleotides of 152-1085, GenBank accession number NM_182993.2). The specificity of this riboprobe for VGLUT1 has been previously established in the mouse (Nakamura et al., 2007) and in both the rat and mouse auditory pathway (Ito et al., 2011). Briefly, this sequence for mouse VGLUT1 is $96 \%$ homologous to the corresponding rat cDNA, while very low for other members of the VGLUT family. The mouse VGLUT1 riboprobe shares only $75 \%$ and $71 \%$ homology with the mouse VGLUT2 and VGLUT3 mRNA, respectively. As a result, expres- sion of this VGLUT1 probe is totally absent in the rat inferior colliculus and other structures that lack VGLUT1 expression in mouse (Ito et al., 2011).

In a subset $(N=6)$ of the brains that received dual retrograde tracer injections, neurons expressing VGLUT1 mRNA were visualized via in situ hybridization in a separate series of tissue as described previously (Ito et al., 2011). Sections in this series were enhanced with silver to visualize neurons retrogradely labeled with CTBG that was injected into either A1 


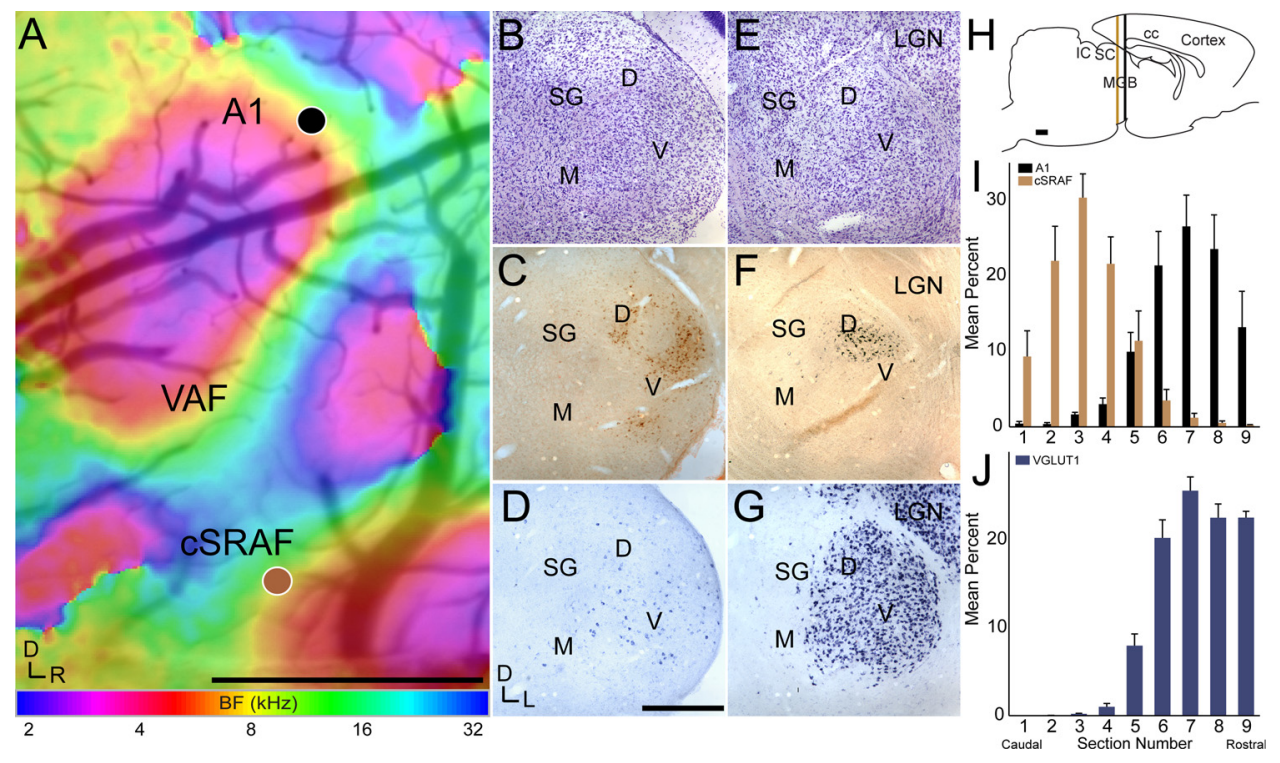

Figure 3. Optical intrinsic imaging, tract tracing, and in situ hybridization distinctions between frequency-organized auditory cortical fields A1 and CSRAF. $A$, Intrinsic imaging map with a surface blood vessel overlay indicates the BF-matched positions for injection sites (colored circles) into A1 and cSRAF. $\boldsymbol{B}-\boldsymbol{D}$, Adjacent sections from caudal MGB ( $-5.98 \mathrm{~mm}$ from bregma) processed for NissI $(\boldsymbol{B})$, CTB, CTBG (C) and VGLUT1/CTBG (D). E-G, Adjacent sections from rostral MGB ( $-5.34 \mathrm{~mm}$ from bregma) processed for Nissl (E), CTB, CTBG (F), and VGLUT1/CTBG (G). Data illustrated in $\boldsymbol{A}-\boldsymbol{G}$ are from the same animal. $\boldsymbol{H}$, Sagittal drawing indicating the approximate positions of sections shown in $\boldsymbol{C}$ and $\boldsymbol{F}$. I, J, Population summary bar graphs for a caudal-to-rostral series of sections of mean percentage of neurons that project to $A 1$ and $C \operatorname{SRAF}(\boldsymbol{I}, N=8$ animals) and neurons that express VGLUT1 mRNA (J,N $=5$ animals). Error bars indicate SEM. Sections in $C, D$ and $F, G$ are included in our population analysis as sections 3 and 7 , respectively, and have their cytoarchitectonic boundaries drawn in Figure 5, Cand G. Scale bars: $\boldsymbol{A}, 1 \mathrm{~mm} ; \boldsymbol{B}-\boldsymbol{G}, 0.5 \mathrm{~mm} ; \boldsymbol{H}, 1 \mathrm{~mm}$. LGN, Lateral geniculate nucleus; $\mathrm{M}$, medial; D, dorsal; $\mathrm{V}$, ventral and SG, suprageniculate divisions of the medial geniculate body; IC, inferior colliculus; $S \mathrm{SC}$, superior colliculus; cc, corpus callosum.

$(N=4)$, or cSRAF $(N=2)$ using light microscopy (Basbaum, 1989). One experiment (A1 injection) was excluded from colocalization analysis due to poorly visualized retrograde label, and another (cSRAF injection) was excluded from caudal-to-rostral analysis due to having been cut at a different angle, however it was otherwise included in our colocalization analysis.

Quantifying labeled neurons and estimating MGB division borders. All labeled neurons (CTB, CTBG, CTB/CTBG, VGLUT1 and VGLUT1/ CTBG) positions and total counts were measured using Stereo Investigator (MBF Bioscience) for a series of sections through the MGB. Soma position corresponded to the nucleolus center as measured with a $100 \times$ objective lens. Labeled neurons were counted if they contained a single distinct nucleolus, and colocalization was identified as neurons containing the black granular reaction product of silver enhanced CTBG confined within the boundaries of immunohistochemical (DAB, brown) or in situ hybridization (NBT/BCIP, blue) reaction product. Data were collected from noncontiguous sections that were separated by at least 160 or $200 \mu \mathrm{m}$, although alternate columns were substituted in the case of damaged or missing sections. For each case, the section corresponding to the bregma position of $-6.30 \mathrm{~mm}$ was identified and coordinates for all other sections are relative to that reference in $160 \mu \mathrm{m}$ increments. Sections separated by $200 \mu \mathrm{m}$ were placed into the nearest bin. Stereo Investigator data were imported into MATLAB to generate cell position plots.

Borders of MGBv, dorsal (MGBd), medial (MGBm), and suprageniculate (SG) nuclei of MGB were based on Nissl-, immuno-, and in situ hybridization-reacted sections, and previous studies in the MGB (LeDoux et al., 1985; Clerici and Coleman, 1990; Paxinos and Watson, 1997; Winer et al., 1999a,b). Quantification of cell counts in each MGB division was performed in MATLAB and measured using cell position plots aligned and overlaid with manually drawn cytoarchitectonic divisions. An automatic routine grouped individual cells into the appropriate nuclear division based on their location within cytoarchitectonic MGB division borders. CTB-, CTBG-, CTB/CTBG-, VGLUT1-, and VGLUT1/CTBG-labeled neurons were analyzed separately from each other.

To control for spurious single-labeled neuron "outliers" in the spatial distributions of labeled neurons, additional bootstrapping statistics were computed for each section using a modified version of our previous technique (Storace et al., 2010, 2011). One thousand random cell distributions were generated from each MATLAB cell position plot marked within a $1500 \times 1500 \mu \mathrm{m}^{2}$ area of the MGB. Each sample was divided into $60 \times 60 \mu \mathrm{m}^{2}$ bins to generate two-dimensional density histograms. The mean bootstrapped histogram was computed and bins were selected for further analysis if they qualified as significant by being three or more SDs above the overall mean count for the section. The areal boundaries of significant bins were marked with a contour, and drawn onto the original cell position plots. Additional cell count measurements were made based on whether labeled neurons were located within these contours. These contours selected for $69.5 \%$ of the original plotted neurons within the boundaries of the MGBv, MGBd, MGBm, and SG subdivisions of the MGB. Original cell counts for neurons projecting to A1 and cSRAF were 3485 and 3781, respectively, and bootstrapped counts were 2533 and 2523 , respectively. The areal pattern of significant clusters was also analyzed using the same coefficient of variation analysis and significance criterion described previously but not included in detail here, and provided additional support for our cytoarchitectonic boundaries in this and prior studies (Polley et al., 2007; Storace et al., 2010, 2011).

\section{Results}

Glutamate transporter types covary with response magnitude and timing (Fremeau et al., 2004b); hence, we examined the possibility that A1 and cSRAF have distinctions in these two response properties (Fig. 1). Frequency organization and regional boundaries of A1 and CSRAF were determined by mapping the BF responses to tones using Fourier optical imaging of intrinsic metabolic signals (Materials and Methods; Fig. 1A). Subsequently, multiunit responses to noise were recorded in both cortical regions, and the time course of the PSTH was fit with an $\alpha$ function as shown for PSTH responses from a site in A1 (black) and a site in cSRAF (gray, Fig. $1 B$ ). Response magnitudes and slope were greater in A1 than CSRAF, and the response delays were shorter (Fig. $1 C-E$ ). Furthermore, the time or delay to peak response increased with dorsal to ventral shifts in cortical positions from A1 to CSRAF (Fig. $1 F$ ). These results indicate 


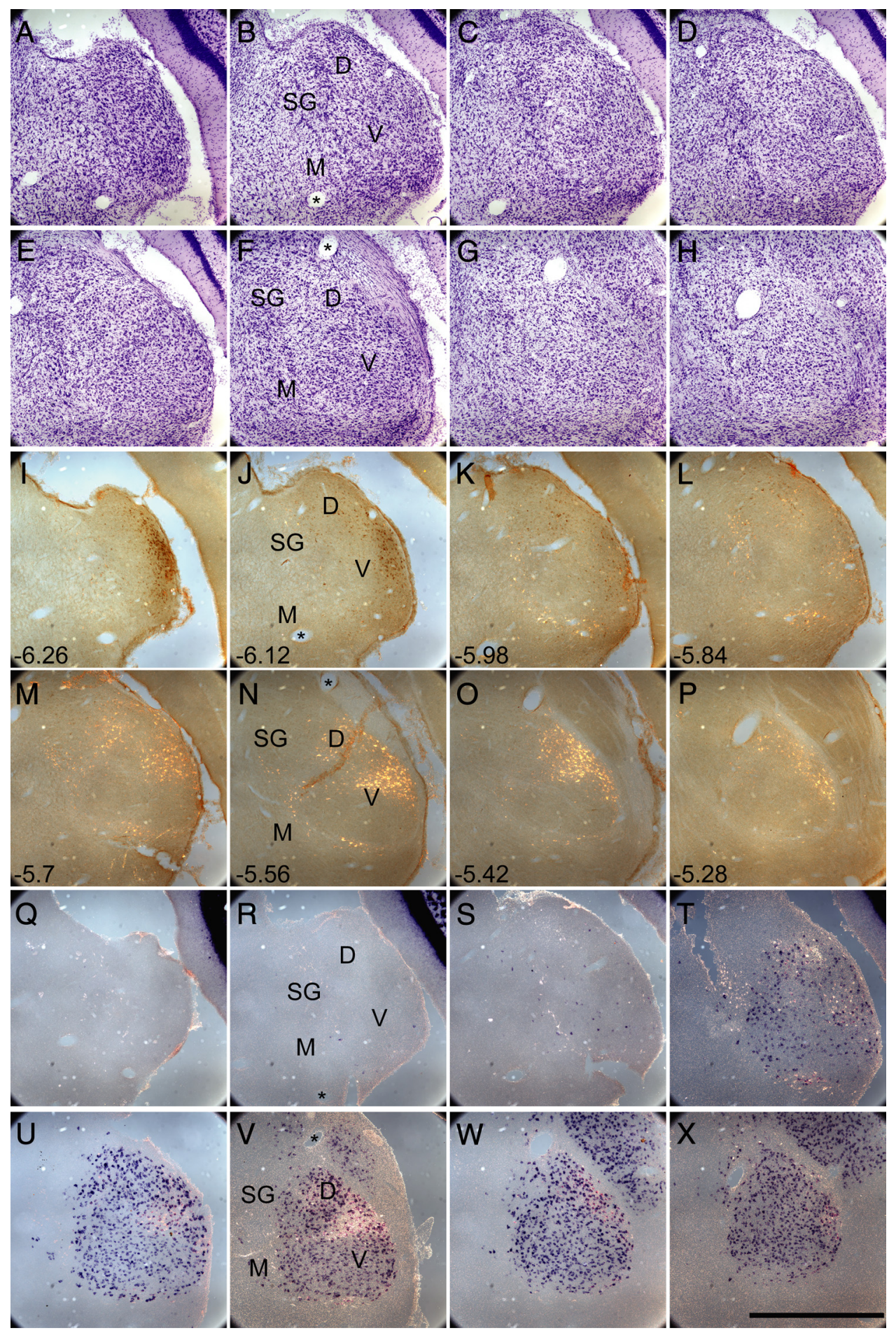

Figure 4. Photomicrographs of three adjacent series of sections from the same animal in $160 \mu \mathrm{m}$ caudal-to-rostral steps through the MGB demonstrate the caudal-rostral distribution of neurons that project to $A 1$ and CSRAF, and those that express VGLUT1 mRNA. $\boldsymbol{A}-\boldsymbol{H}$, Nissl-stained sections demonstrate MGB cytoarchitecture along the caudal-rostral axis. I-P, CTB and CTBG double-reacted sections demonstrate that neurons that project to $\operatorname{SRAF}$ (brown) and A1 (gold) are found in the caudal and rostral parts of MGB, respectively. Numbers indicate approximate distance (mm) from bregma. Q- $\boldsymbol{X}$, Sections reacted for both CTBG and VGLUT1 mRNA demonstrate the caudal-to-rostral increase in the total number of neurons expressing VGLUT1 mRNA (blue), and the areal pattern of neurons that also project to A1 (gold). Scale bar, $1 \mathrm{~mm}$.

that $\mathrm{Al}$ and cSRAF have distinct frequency organization and sound response properties.

The pattern and relative density of VGLUT2 protein expression has not been demonstrated for physiologically identified Al or cSRAF in the rat. Here we used optical imaging to map the frequency response organization and to guide injections of fluorescent beads into frequency-matched regions of A1 and cSRAF (Fig. 2A). The brains were subsequently processed to examine the laminar organization of cortical cell bodies and VGLUT2 protein-expressing terminal fields in A1 and cSRAF. Both cortical fields showed a similar Nissl profile (Fig. $2 B, E$ ) and a high density of VGLUT2 protein expression in the middle cell layers (Fig. 2C,F). In contrast, VGLUT2 protein expression dropped markedly in the transition between rSRAF and the ventral "auditory association" area, Te3 (Fig. 2C, Te3 vs rSRAF). 

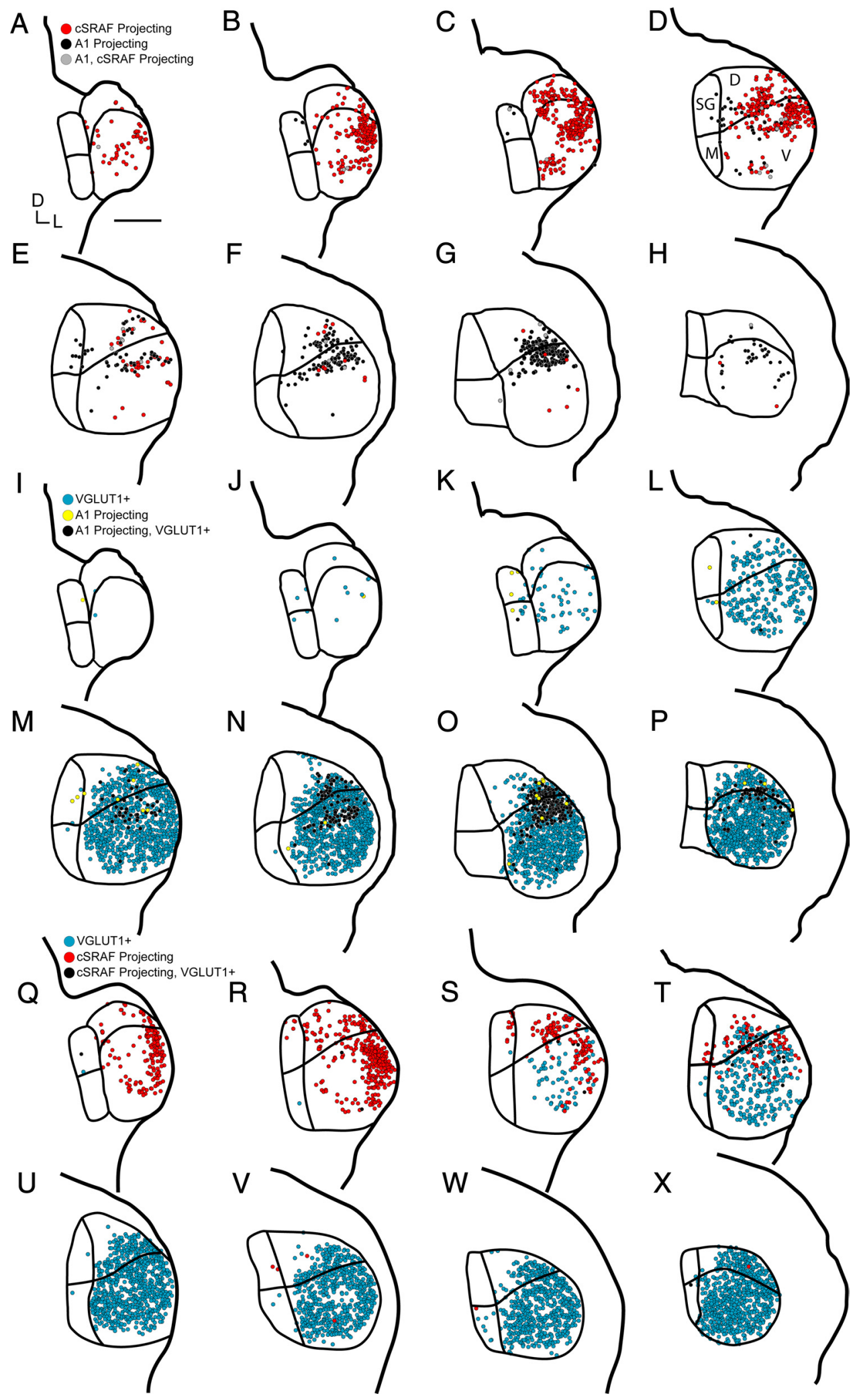

Figure 5. Areal plots of MGB neurons that project to $A 1$ and CSRAF and neurons that express VGLUT1 mRNA. A-H, MGB division boundaries and cell position plots of sections double-reacted for CTB and CTBG showing the caudal-to-rostral separation of neurons that project to CSRAF (red) and A1 (black). I-P, MGB division boundaries and cell position plots of sections double-reacted for retrograde tracer injections into A1 (CTBG), and processed for VGLUT1 mRNA using in situ hybridization demonstrate neurons expressing VGLUT1 mRNA (blue), neurons that project to A1 that express VGLUT1 mRNA (black), and those that do not express the gene (yellow). $\mathbf{Q}-\boldsymbol{X}$, MGB division boundaries and cell position plots of sections processed to reveal retrograde tracer following injections into CSRAF (CTBG), and double-reacted for VGLUT1 mRNA using in situ hybridization demonstrate neurons that express VGLUT1 mRNA (blue), neurons that project to cSRAF that express VGLUT1 mRNA (black), and those that do not express the gene (red). Neurons expressing VGLUT1 mRNA were primarily found in MGBv and MGBd, and the number of VGLUT1+ neurons increased from caudal-to-rostral MGB. The majority of neurons that project to A1 also expressed VGLUT1 mRNA (I-P, black), whereas most neurons that project to cSRAF do not express the gene ( $\mathbf{Q}-\boldsymbol{X}$, red). Sections in $\mathbf{A}-\mathbf{P}$ and $\mathbf{Q}-\boldsymbol{X}$ are from two different animals. Scale bar, $0.5 \mathrm{~mm}$. 
A plexus of VGLUT2 putative terminals with bouton-like swellings was evident at high magnification in A1 and cSRAF (Fig. 2D,G). This resembles that seen in other auditory and sensory cortices (Nahmani and Erisir, 2005; Graziano et al., 2008; Hackett and de la Mothe, 2009) and suggests that VGLUT2 protein expression alone does not readily distinguish the thalamocortical pathways to frequency-organized fields, A1 versus cSRAF.

We examined thalamic input sources to A1 and cSRAF as a potential source of response property differences in the two fields (Fig. 3 ). Retrograde tracers CTB and CTBG were deposited in layer IV of frequency-matched $8 \mathrm{kHz}$ contours in $\mathrm{A} 1$ and cSRAF (Fig. 3A). Nissl-stained sections illustrate the distribution of all MGB neurons in caudal (Fig. 3B) and rostral (Fig. 3E) MGB from one dual-injected brain hemisphere. Adjacent sections processed to visualize both tracers illustrate independent and complementary caudal and rostral locations of the subsets of thalamic neurons projecting to cSRAF (Fig. $3 C$ ) and A1 (Fig. 3F), respectively. This caudal-to-rostral organization is demonstrated in a series of photomicrographs of sections through the MGB in a separate experiment (Fig. $4 A-H$, Nissl; $I-P$, immunoreacted). The complementary caudal-to-rostral distribution is also evident in the population average of retrogradely labeled cell counts in MGB for each section in a series of 9 sections per animal in 8 animals (Fig. 3I). While the entire division of $\mathrm{MGBv}$ contained Nissl-stained neurons (Figs. $3 B, E, 4 A-H$ ), retrogradely labeled cells only occupied a small portion of the MGBv area in each section (Fig. 4I-P). Rostral MGBv neurons that lack retrograde tracer and yet stain for Nissl in adjacent sections (Figs. $3 E, F, 4 G, O$ ) likely project to neighboring isofrequency contours in A1, as demonstrated in a previous study with multiple retrograde tracers into 4 neighboring cortical isofrequency contours that span over 3 octaves of representation (Storace et al., 2011). An average of 8.5 ( \pm 3.2 ) neurons per animal were double-labeled for both retrograde tracers, accounting for $<1 \%$ of all labeled neurons across all animals. These data establish that frequency-matched regions of A1 and cSRAF receive input from separate populations of thalamic neurons.

Neurons located outside of the MGBv project more densely to auditory cortical fields outside of A1 in the rat (Arnault and Roger, 1990). Hence, we considered the possibility that the response property differences in CSRAF could be attributed to input from these non-MGBv thalamic divisions. To address this question, the positions of retrogradely labeled neurons were plotted and analyzed for a caudal-to-rostral series of sections spanning $\sim 1.5 \mathrm{~mm}$, as illustrated for one dual-injected brain hemisphere (Fig. 5A-H). The mean number and percentage of retrogradely labeled neurons in the MGB (Fig. $6 A, B$, respectively) was similar for injections in A1 (black bars) or cSRAF (gray bars). Most (70\%) of the labeled thalamic neurons with injections into A1 and cSRAF combined were located in $\mathrm{MGBv}$, with the remaining located in non-MGBv divisions. Slightly more MGBd neurons were connected to cSRAF; whereas, slightly more SG neurons were connected to A1 (Fig. 6A,B). This confirms that $\mathrm{MGBv}$ is the primary source of connections to the target layer of both cortical fields as it typical of a core field, and that non-MGBv connections may serve to distinguish the two thalamocortical pathways.

Previously, VGLUT1 mRNA expression was seen in the cell bodies of the majority of neurons in the MGBv of the rat (Ito et al., 2011). Here, we quantified this pattern of gene expression and asked whether the thalamic neurons expressing VGLUT1 projected to A1 as well as cSRAF. VGLUT1 gene expression was minimal in the caudal portions of the MGBv, the area that contained the greatest number of neurons that projected to cSRAF (Fig. 3D). In contrast, neurons expressing the VGLUT1 gene were
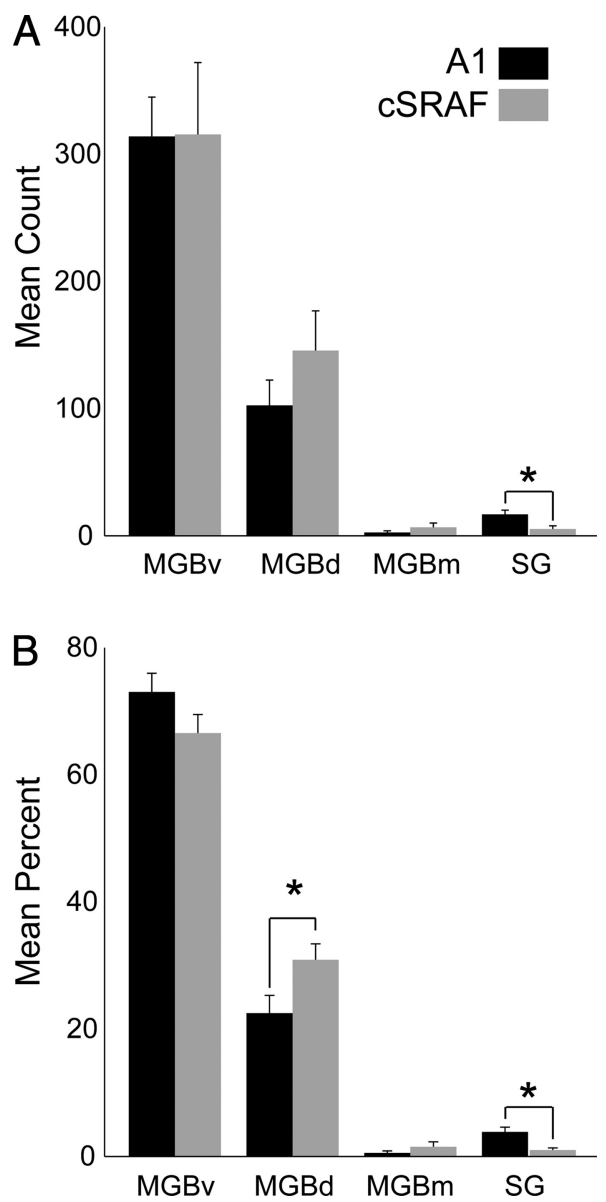

Figure 6. Injections into frequency-matched $A 1$ and CSRAF labeled similar numbers $(\boldsymbol{A})$ and percentages $(\boldsymbol{B})$ in each MGB division, with the majority located in the MGBv. A slightly higher percentage of MGBd neurons projected to CSRAF than to $A 1\left(t_{(14)}=-2.3, p=0.04\right)$, and slightly more $S G$ neurons projected to $A 1$ than to $\operatorname{CSRAF}\left(t_{(14)}=3.4, p<0.05\right)$. Data are means and SEM from eight animals. ${ }^{*} p<0.05$ using a two-tailed $t$ test.

highly concentrated in the rostral portion of MGBv (Fig. 3G), the area that contained the greatest number of neurons that projected to A1. The number of neurons projecting to A1 increased $\sim 100$-fold, on average, over the $\sim 1.5 \mathrm{~mm}$ distance from the caudal to rostral MGB (Fig. 3I). Likewise, the number of VGLUT1 + neurons increased by $\sim 1000$-fold, on average, over the same distance (Fig. $3 J$ ). The caudal-rostral transition of VGLUT1 mRNA expression is demonstrated in three different animals as photomicrographs (Fig. 4Q-X) and two series of plotted sections (Fig. $5 I-P, Q-X$ ). The caudal-to-rostral relationship between MGB neurons that project to $A 1$, and those that express VGLUT1 mRNA is further supported by a significant positive correlation $(r=0.93, p<0.001)$, and the presence of $<2 \%$ of VGLUT1 + neurons in the three sections containing most cells $(75 \%)$ that projected to cSRAF (Fig. 3I, J, sections 2-4). These data confirm glutamate transporter gene expression changes along the caudal-to-rostral axis of the MGBv.

To determine whether the population of neurons that project to A1 versus cSRAF could be distinguished by the presence of VGLUT1 mRNA, tissue was dual processed to reveal neurons containing retrograde tracer and expressing VGLUT1 mRNA. Dual processed sections are shown from two animals in caudal (Fig. $7 A, B$ ) and rostral (Fig. 7C,D) MGB following injections into cSRAF (Fig. $7 A, C$ ) or frequency-matched A1 (Fig. $7 B, D$ ). 

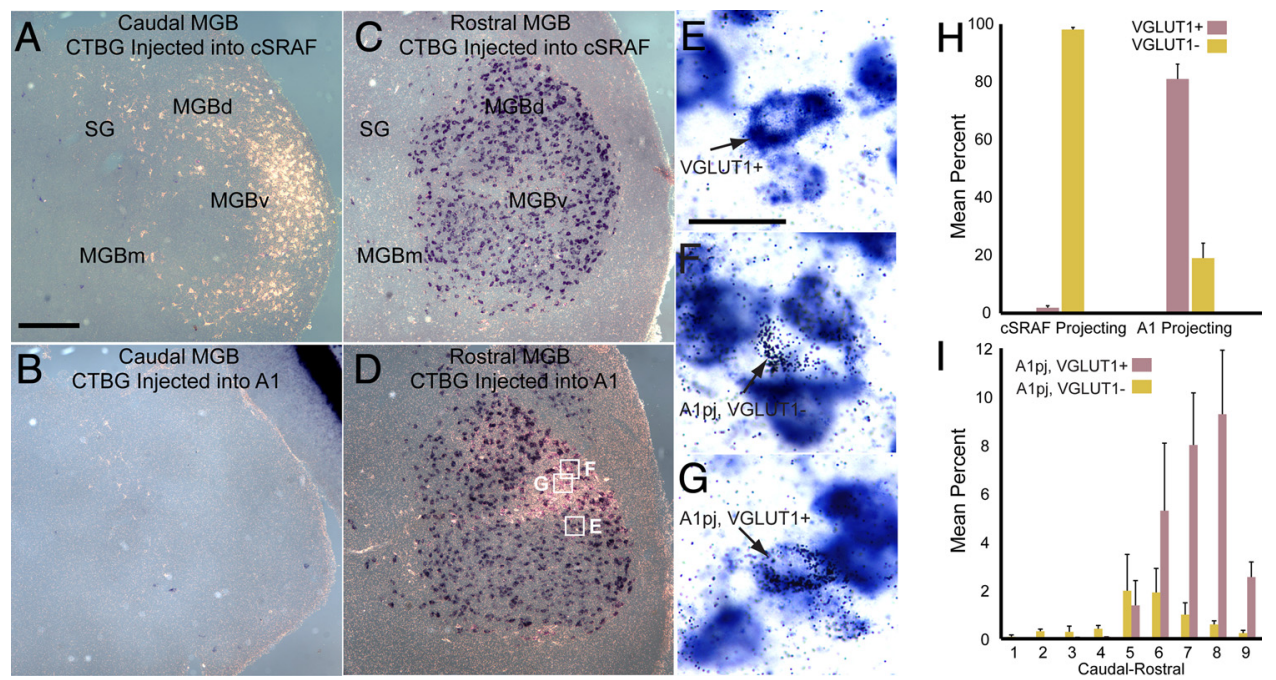

Figure 7. Neurons that project to A1 and CSRAF can be distinguished by the expression of VGLUT1 mRNA. Brain sections were dual-processed to reveal retrograde tracer and in situ hybridization for VGLUT1 mRNA. A-D, Photomicrographs of dual-processed tissue. A, C, An example of label patterns following injection into CSRAF in one animal. Neurons in caudal MGB were densely labeled with retrograde tracer $(\boldsymbol{A})$ but did not express VGLUT1 mRNA as evidenced by the lack of purple reaction product. VGLUT1 expressing neurons (purple) in rostral MGB of the same animal lacked retrograde label $(\boldsymbol{C}) \cdot \boldsymbol{B}, \boldsymbol{D}$, An example of label patterns following injection into $A 1$ in a second animal. Neurons in caudal MGB were not labeled with retrograde tracer (B), nor did they express VGLUT1 mRNA. In contrast, neurons in rostral MGB expressed VGLUT1 mRNA, and were double-labeled with retrograde tracer following injections into A1 (D). $\boldsymbol{E}$-G, High-magnification photomicrographs of the three types of labeled neurons taken from the white boxes in $\boldsymbol{D}$. $\boldsymbol{E}$, neurons that expressed VGLUT1 mRNA; $\boldsymbol{F}$, retrogradely labeled neurons; and $\boldsymbol{G}$, retrogradely labeled neurons that expressed VGLUT1 mRNA. $\boldsymbol{H}$, I, Summary of data from 2 and 3 animals injected in CSRAF and A1, respectively. $\boldsymbol{H}$, Most labeled neurons that projected to A1 expressed VGLUT1 mRNA (81 $\pm 5 \%$ ), whereas most labeled neurons that projected to CSRAF did not $(98 \pm 0.8 \%)$. $I$, The mean percentage of A1 projecting neurons positive for VGLUT1 mRNA was highest in the rostral part of the MGB. Error bars indicate SEM. Scale bars: $\boldsymbol{A}, 0.25 \mathrm{~mm} ; \boldsymbol{B}-\boldsymbol{E}, 0.25 \mu \mathrm{m}$. A1pj, A1 projecting.

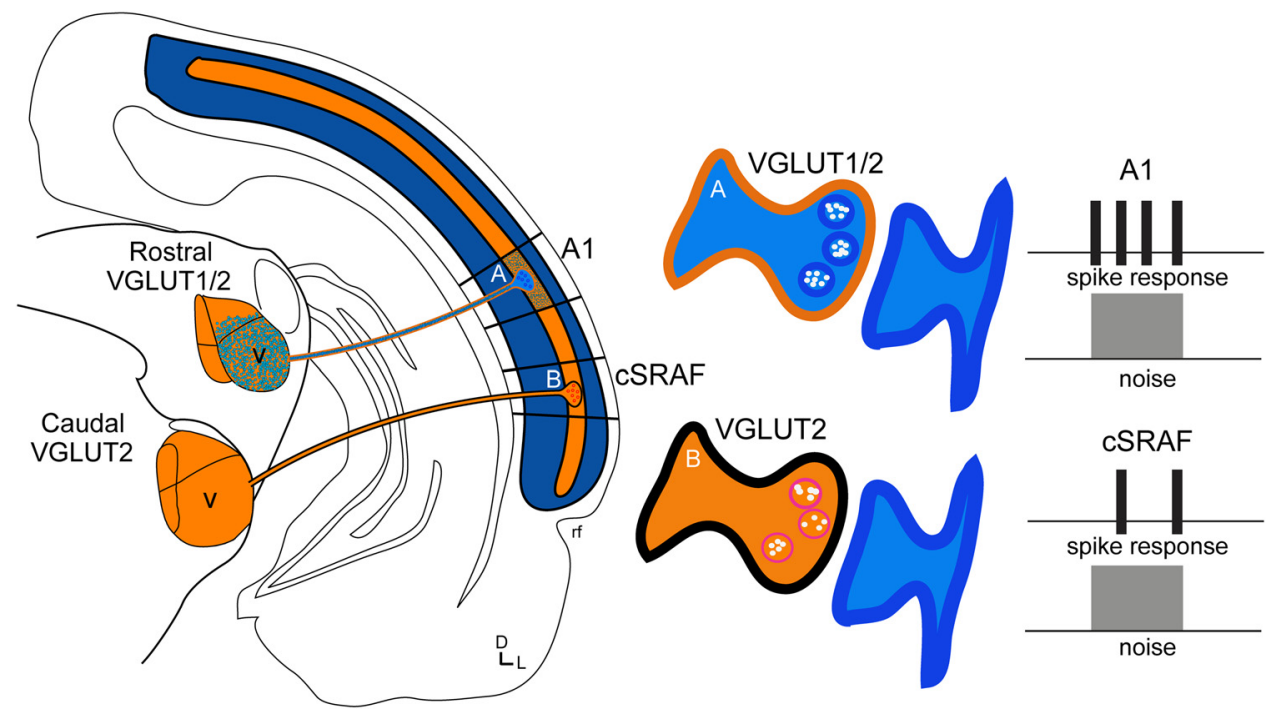

Figure 8. A parallel thalamocortical pathway organization distinguished by thalamic connections and VGLUT1 mRNA expression patterns as demonstrated in this study indicates novel organization principles. $\boldsymbol{A}$, Neurons in rostral MGB that express VGLUT1 gene terminate in A1 where recordings demonstrate short-latency, high spike rates to noise. $\boldsymbol{B}$, Neurons in caudal MGB that do not express the VGLUT1 gene terminate in CSRAF where recordings demonstrate longer-latency, lower spike rate responses to noise. The organization scheme illustrated is supported by data in the present study that finds a dense terminal band of VGLUT2 protein in both A1 and CSRAF, and a systematic change in cortical connections and VGLUT1 expression pattern differences between caudal versus rostral MGB. This scheme is consistent with the hypothesis that there exists a dense VGLUT1 protein innervation to layer IV of A1, but not CSRAF.

Neurons in caudal MGB had low VGLUT1 mRNA expression, and were densely connected to CSRAF (Fig. 7A), but not to A1 (Fig. $7 B$ ). In contrast, neurons in rostral MGB had high VGLUT1 mRNA expression, and were densely connected to A1 (Fig. 7D), but not to cSRAF (Fig. 7C). High-magnification images reveal neurons that expressed VGLUT1 mRNA only (Fig. 7E), neurons labeled with retrograde tracer only (Fig. 7F, CTBG), and neurons double-labeled with VGLUT1 mRNA and retrograde tracer (Fig. 7G).
Quantification of labeling patterns in 3 animals revealed that $81 \%$ ( \pm 5.2$)$ of the neurons that projected to A1 expressed VGLUT1 mRNA (Fig. 7H), and were positioned in rostral MGBv (Fig. 7I). In contrast, quantification of labeling patterns in 2 animals revealed that $<3 \%$ of the neurons that projected to cSRAF expressed VGLUT1 mRNA (Fig. 7H). The caudal-rostral distributions for experiments double-reacted for VGLUT1 mRNA, and silver enhanced for tracer injection into A1 or cSRAF are plotted (Fig. 5I-P,Q-X, respectively). These results indicate that the majority of neurons pro- 
jecting to the $8 \mathrm{kHz}$ contour of A1 express VGLUT1, while those that project to frequency-matched cSRAF do not.

\section{Discussion}

It is common for mammals including human to have multiple cortical areas with distinct sound frequency response organization and processing and yet the etiology and purpose of these parallel representations is still not fully known (Schreiner et al., 2000; Schreiner and Winer, 2007; Hackett, 2011). Here we find the MGBv division of thalamus is the primary source of connections to layer IV for two frequency-organized fields, $\mathrm{Al}$ and CSRAF, in the rat (Fig. 6). VGLUT2 gene expression is high throughout the caudal-to-rostral extent of MGBv (Barroso-Chinea et al., 2007; Ito et al., 2011) and a high VGLUT2 protein expression in cortical layers III and IV (Fig. 2) suggests both thalamocortical pathways use glutamate as a neurotransmitter (Fig. 8). However, we find that A1 and CSRAF have completely independent representations of sound frequency (Fig. $1 A$ ) and are connected to rostral and caudal halves of the MGBv, respectively, with $<1 \%$ of MGBv neurons projecting to both regions. Furthermore, the majority (81\%) of rostral MGBv neurons that project to A1 express the VGLUT1 gene, whereas few (3\%) of the caudal MGBv neurons that project to the frequency-matched region of CSRAF express the VGLUT1 gene. Together this indicates functionally independent and parallel $\mathrm{MGBv}$ pathways to frequency-matched regions of neighboring frequency-organized auditory cortical fields (Fig. 8, caudal vs rostral pathways).

This study offers a promising new approach to identifying functionally distinct auditory pathways and potential molecular markers for specialization of auditory pathways across development and mammalian species. VGLUT2 gene expression is observed early in development throughout auditory thalamus and expressed minimally in cortical neurons (Fremeau et al., 2004b; Hackett et al., 2011; Ito et al., 2011) and corresponding VGLUT2 protein expression is likely situated on MGB terminals synapsing in cortex. In primate, the density of VGLUT2 protein expression is higher in A1 than surrounding belt and parabelt fields (Hackett and de la Mothe, 2009). Similarly, in the rat we find a higher density of VGLUT2 protein expression in A1 compared with the non-frequency-organized auditory "association" field Te3 located dorsal to the rhinal fissure and ventral to SRAF (Fig. 2C). A finer grain quantitative analysis may reveal additional laminar pattern differences for VGLUT2 protein expression in the rat. In primate, convergent inputs from non-MGBv (VGLUT2 expressing) thalamic pathways is another source of differences across cortical fields (Kaas and Hackett, 2000). Here, we find similar percentages and absolute numbers of MGBv neurons projecting to A1 and CSRAF (Fig. 6). Slightly more MGBd neurons projected to cSRAF; whereas, slightly more SG neurons projected to A1 (Fig. 6). The non-MGB connections with auditory cortex may be underestimated with the approach used here, as deposits were centered in layer IV, not layer III where MGBd synapses are highly concentrated (Huang and Winer, 2000; Smith et al., 2012). The highest density of VGLUT1 gene expression is in rostral MGBv in adult rat and mouse, indicating phenotypic conservation across rodent species (Ito et al., 2011). Preliminary studies suggest a possible phylogenetic difference and lack of VGLUT1 expression in MGBv of adult primate (Ito and Takada, 2011). The latter may be related to other phylogenetic differences including higher concentrations of inhibitory interneurons in MGBv (Winer et al., 1996; Peruzzi et al., 1997) and higher resolution of frequency responses to sound in cats and primates versus rats (Schreiner et al., 2000; Cheung et al., 2001; Read et al., 2002; Polley et al., 2007). These and many other factors likely contribute to variation in vesicular transporter gene and protein expression patterns during development as well as across species.
What might be the physiologic advantage to expressing two glutamate transporters in pathways to A1? Prior studies suggest coexpression of VGLUT1 and VGLUT2 allow for high response magnitudes and rates at corresponding synapses. VGLUT2 is expressed early in development and current theories suggest the highly reliable glutamate release associated with VGLUT2 is needed to establish thalamocortical sensory pathways (Nakamura et al., 2005; Liguz-Lecznar and Skangiel-Kramska, 2007). The sensitivity and magnitude of thalamocortical sensory responses increase during development (Zhang et al., 2001). Corresponding increased neural activity shapes the VGLUT1 and VGLUT2 expression patterns in several systems, indicating a refinement in synaptic processing (De Gois et al., 2005; Yoshida et al., 2009). In adult rats, the majority of MGBv cells that express VGLUT1 also coexpress VGLUT2 (Ito et al., 2011); hence, the population of cells identified here as projecting to A1 and expressing VGLUT1, likely coexpresses VGLUT2 (Fig. 8A). VGLUT1 and VGLUT2 transporters can colocalize to the same synaptic vesicles (Herzog et al., 2006). Furthermore, VGLUT1 and VGLUT2 bind to distinct vesicle trafficking proteins (De Gois et al., 2006), and the combined expression of both transporters results in more transmitter per vesicle, bigger and faster postsynaptic responses and higher spike rate output (Fremeau et al., 2004b; Wojcik et al., 2004; Wilson et al., 2005). In theory, the higher magnitude and faster time course of responses in Al that we report here could require the coexpression of VGLUT1 and VGLUT2 at corresponding thalamocortical synapses (Fig. 8). The present study demonstrates a gene expression pattern that marks differences in parallel core ascending auditory pathways, and calls for future studies to more directly relate glutamate transporter type to dynamic properties of cortical sound processing.

\section{References}

Arnault P, Roger M (1990) Ventral temporal cortex in the rat: connections of secondary auditory areas Te2 and Te3. J Comp Neurol 302:110-123.

Barroso-Chinea P, Castle M, Aymerich MS, Pérez-Manso M, Erro E, Tuñon T, Lanciego JL (2007) Expression of the mRNAs encoding for the vesicular glutamate transporters 1 and 2 in the rat thalamus. J Comp Neurol 501:703-715.

Basbaum AI (1989) A rapid and simple silver enhancement procedure for ultrastructural localization of the retrograde tracer WGAapoHRP-Au and its use in double-label studies with post-embedding immunocytochemistry. J Histochem Cytochem 37:1811-1815.

Budinger E, Scheich H (2009) Anatomical connections suitable for the direct processing of neuronal information of different modalities via the rodent primary auditory cortex. Hear Res 258:16-27.

Cant NB, Benson CG (2007) Multiple topographically organized projections connect the central nucleus of the inferior colliculus to the ventral division of the medial geniculate nucleus in the gerbil, Meriones unguiculatus. J Comp Neurol 503:432-453.

Cheung SW, Bedenbaugh PH, Nagarajan SS, Schreiner CE (2001) Functional organization of squirrel monkey primary auditory cortex: responses to pure tones. J Neurophysiol 85:1732-1749.

Clerici WJ, Coleman JR (1990) Anatomy of the rat medial geniculate body: I. Cytoarchitecture, myeloarchitecture, and neocortical connectivity. J Comp Neurol 297:14-31.

De Gois S, Schäfer MK, Defamie N, Chen C, Ricci A, Weihe E, Varoqui H, Erickson JD (2005) Homeostatic scaling of vesicular glutamate and GABA transporter expression in rat neocortical circuits. J Neurosci 25: 7121-7133.

De Gois S, Jeanclos E, Morris M, Grewal S, Varoqui H, Erickson JD (2006) Identification of endophilins 1 and 3 as selective binding partners for VGLUT1 and their co-localization in neocortical glutamatergic synapses: implications for vesicular glutamate transporter trafficking and excitatory vesicle formation. Cell Mol Neurobiol 26:679-693.

Donishi T, Kimura A, Okamoto K, Tamai Y (2006) "Ventral" area in the rat auditory cortex: a major auditory field connected with the dorsal division of the medial geniculate body. Neuroscience 141:1553-1567.

Escabí MA, Higgins NC, Galaburda AM, Rosen GD, Read HL (2007) Early 
cortical damage in rat somatosensory cortex alters acoustic feature representation in primary auditory cortex. Neuroscience 150:970-983.

Formisano E, Kim DS, Di Salle F, van de Moortele PF, Ugurbil K, Goebel R (2003) Mirror-symmetric tonotopic maps in human primary auditory cortex. Neuron 40:859-869.

Fremeau RT Jr, Voglmaier S, Seal RP, Edwards RH (2004a) VGLUTs define subsets of excitatory neurons and suggest novel roles for glutamate. Trends Neurosci 27:98-103.

Fremeau RT Jr, Kam K, Qureshi T, Johnson J, Copenhagen DR, Storm-Mathisen J, Chaudhry FA, Nicoll RA, Edwards RH (2004b) Vesicular glutamate transporters 1 and 2 target to functionally distinct synaptic release sites. Science 304:1815-1819.

Graziano A, Liu XB, Murray KD, Jones EG (2008) Vesicular glutamate transporters define two sets of glutamatergic afferents to the somatosensory thalamus and two thalamocortical projections in the mouse. J Comp Neurol 507:1258-1276.

Hackett TA (2011) Information flow in the auditory cortical network. Hear Res 271:133-146.

Hackett TA, de la Mothe LA (2009) Regional and laminar distribution of the vesicular glutamate transporter, VGluT2, in the macaque monkey auditory cortex. J Chem Neuroanat 38:106-116.

Hackett TA, Takahata T, Balaram P (2011) VGLUT1 and VGLUT2 mRNA expression in the primate auditory pathway. Hear Res 274:129-141.

Herzog E, Takamori S, Jahn R, Brose N, Wojcik SM (2006) Synaptic and vesicular co-localization of the glutamate transporters VGLUT1 and VGLUT2 in the mouse hippocampus. J Neurochem 99:1011-1018.

Higgins NC, Storace DA, Escabí MA, Read HL (2010) Specialization of binaural responses in ventral auditory cortices. J Neurosci 30:14522-14532.

Huang CL, Winer JA (2000) Auditory thalamocortical projections in the cat: laminar and areal patterns of input. J Comp Neurol 427:302-331.

Ito T, Takada M (2011) Distribution of glutamatergic, GABAergic, and glycinergic neurons in the auditory brainstem of Japanese macaque (Macaca fuscata). Neurosci Res 71 [Suppl]:e152.

Ito T, Bishop DC, Oliver DL (2011) Expression of glutamate and inhibitory amino acid vesicular transporters in the rodent auditory brainstem. J Comp Neurol 519:316-340.

Kaas JH, Hackett TA (2000) Subdivisions of auditory cortex and processing streams in primates. Proc Natl Acad Sci U S A 97:11793-11799.

Kalatsky VA, Polley DB, Merzenich MM, Schreiner CE, Stryker MP (2005) Fine functional organization of auditory cortex revealed by Fourier optical imaging. Proc Natl Acad Sci U S A 102:13325-13330.

Kaneko T, Fujiyama F, Hioki H (2002) Immunohistochemical localization of candidates for vesicular glutamate transporters in the rat brain. J Comp Neurol 444:39-62.

LeDoux JE, Ruggiero DA, Reis DJ (1985) Projections to the subcortical forebrain from anatomically defined regions of the medial geniculate body in the rat. J Comp Neurol 242:182-213.

Liguz-Lecznar M, Skangiel-Kramska J (2007) Vesicular glutamate transporters VGLUT1 and VGLUT2 in the developing mouse barrel cortex. Int J Dev Neurosci 25:107-114.

Lomber SG, Malhotra S (2008) Double dissociation of 'what' and 'where' processing in auditory cortex. Nat Neurosci 11:609-616.

Morest DK (1964) The neuronal architecture of the medial geniculate body of the cat. J Anat 98:611-630.

Nahmani M, Erisir A (2005) VGluT2 immunochemistry identifies thalamocortical terminals in layer 4 of adult and developing visual cortex. J Comp Neurol 484:458-473.

Nakamura K, Hioki H, Fujiyama F, Kaneko T (2005) Postnatal changes of vesicular glutamate transporter (VGluT) 1 and VGluT2 immunoreactivities and their colocalization in the mouse forebrain. J Comp Neurol 492:263-288.

Nakamura K, Watakabe A, Hioki H, Fujiyama F, Tanaka Y, Yamamori T, Kaneko T (2007) Transiently increased colocalization of vesicular glu- tamate transporters 1 and 2 at single axon terminals during postnatal development of mouse neocortex: a quantitative analysis with correlation coefficient. Eur J Neurosci 26:3054-3067.

Nodal FR, Kacelnik O, Bajo VM, Bizley JK, Moore DR, King AJ (2010) Lesions of the auditory cortex impair azimuthal sound localization and its recalibration in ferrets. J Neurophysiol 103:1209-1225.

Paxinos G, Watson C (1997) Rat brain in stereotaxic coordinates. San Diego: Academic.

Peruzzi D, Bartlett E, Smith PH, Oliver DL (1997) A monosynaptic GABAergic input from the inferior colliculus to the medial geniculate body in rat. J Neurosci 17:3766-3777.

Polley DB, Steinberg EE, Merzenich MM (2006) Perceptual learning directs auditory cortical map reorganization through top-down influences. J Neurosci 26:4970-4982.

Polley DB, Read HL, Storace DA, Merzenich MM (2007) Multiparametric auditory receptive field organization across five cortical fields in the albino rat. J Neurophysiol 97:3621-3638.

Read HL, Winer JA, Schreiner CE (2002) Functional architecture of auditory cortex. Curr Opin Neurobiol 12:433-440.

Redies H, Brandner S, Creutzfeldt OD (1989) Anatomy of the auditory thalamocortical system of the guinea pig. J Comp Neurol 282:489-511.

Rodrigues-Dagaeff C, Simm G, De Ribaupierre Y, Villa A, De Ribaupierre F, Rouiller EM (1989) Functional organization of the ventral division of the medial geniculate body of the cat: evidence for a rostro-caudal gradient of response properties and cortical projections. Hear Res 39:103-125.

Schreiner CE, Winer JA (2007) Auditory cortex mapmaking: principles, projections, and plasticity. Neuron 56:356-365.

Schreiner CE, Read HL, Sutter ML (2000) Modular organization of frequency integration in primary auditory cortex. Annu Rev Neurosci 23:501-529.

Smith PH, Uhlrich DJ, Manning KA, Banks MI (2012) Thalamocortical projections to rat auditory cortex from the ventral and dorsal divisions of the medial geniculate nucleus. J Comp Neurol 520:34-51.

Storace DA, Higgins NC, Read HL (2010) Thalamic label patterns suggest primary and ventral auditory fields are distinct core regions. J Comp Neurol 518:1630-1646.

Storace DA, Higgins NC, Read HL (2011) Thalamocortical pathway specialization for sound frequency resolution. J Comp Neurol 519:177-193.

Wallace MN, Rutkowski RG, Palmer AR (2000) Identification and localisation of auditory areas in guinea pig cortex. Exp Brain Res 132:445-456.

Wilson NR, Kang J, Hueske EV, Leung T, Varoqui H, Murnick JG, Erickson JD, Liu G (2005) Presynaptic regulation of quantal size by the vesicular glutamate transporter VGLUT1. J Neurosci 25:6221-6234.

Winer JA, Saint Marie RL, Larue DT, Oliver DL (1996) GABAergic feedforward projections from the inferior colliculus to the medial geniculate body. Proc Natl Acad Sci U S A 93:8005-8010.

Winer JA, Kelly JB, Larue DT (1999a) Neural architecture of the rat medial geniculate body. Hear Res 130:19-41.

Winer JA, Sally SL, Larue DT, Kelly JB (1999b) Origins of medial geniculate body projections to physiologically defined zones of rat primary auditory cortex. Hear Res 130:42-61.

Winer JA, Miller LM, Lee CC, Schreiner CE (2005) Auditory thalamocortical transformation: structure and function. Trends Neurosci 28:255-263.

Wojcik SM, Rhee JS, Herzog E, Sigler A, Jahn R, Takamori S, Brose N, Rosenmund C (2004) An essential role for vesicular glutamate transporter 1 (VGLUT1) in postnatal development and control of quantal size. Proc Natl Acad Sci U S A 101:7158-7163.

Yoshida M, Satoh T, Nakamura KC, Kaneko T, Hata Y (2009) Cortical activity regulates corticothalamic synapses in dorsal lateral geniculate nucleus of rats. Neurosci Res 64:118-127.

Zhang LI, Bao S, Merzenich MM (2001) Persistent and specific influences of early acoustic environments on primary auditory cortex. Nat Neurosci 4:1123-1130. 